\title{
Avaliação do Processo de atendimento de demandas de produtos de software da Embrapa
}

\author{
Edméia Leonor Pereira de \\ Andrade \\ Embrapa \\ edmeia.andrade@embrapa.br
}

\author{
AngélicaToffano Seidel \\ Calazans \\ Caixa Econômica Federal
}

angelica.calazans@caixa.gov.br

\author{
Káthia Marçal de Oliveira, \\ Adelaide Figueiredo \\ Univ. Católica de Brasília \\ kathia@ucb.br,adelaid@pos.ucb.br
}

\section{Resumo}

A melhoria de processo de software tem recebido mais atenção e visibilidade das organizações e dos profissionais de software desde a década de 90, quando começaram a surgir normas e modelos para melhorar e medir os processos. A medição possibilita a identificação de dados quantitativos e qualitativos e fornece mecanismos para avaliar e melhorar o processo. Esse documento apresenta um relato de experiência com a avaliação do Processo de atendimento de demandas de produto de software em uso na Embrapa através da aplicação do Goal Question-Metrics (GQM).

\section{Palavras-chave}

Processos de software, Medição, Métricas, GQM

\begin{abstract}
Software process improvements have received greater attention and visibility from organizations and software professionals since the $90^{\text {s. }}$. By the beginning of the decade, new normalization and models were developed to measure software process quality. These measurements allowed the identification of quantitative and qualitative data about the process development and defined evaluation mechanisms through which is possible to keep a continuous improvement in software process quality. In this paper, a software process from Embrapa will be appraised through the application of Goal Question Metrics (GQM).
\end{abstract}

\section{Keywords}

Software Process, Measurement, Metrics, GQM

\section{Introdução}

A melhoria de processos de software tem recebido mais atenção e visibilidade da indústria e dos profissionais de software desde a década de 90 , quando começaram a surgir normas e modelos para medir e melhorar os processos de software tais como a norma ISO 12207, modelo SPICE (ISO/IEC 15504) e o modelo CMM (Capability Maturity Model).

Todos estes modelos propõem a definição e/ou melhoria do processo para gerar níveis mais altos de qualidade do produto de software. Porém, para que isso se torne possível, é preciso definir, implantar, medir, analisar e melhorar os processos. A medição possibilita a identificação de dados quantitativos e qualitativos sobre a realidade do processo e fornece 
mecanismos para avaliar e melhorar o processo. A medição se dá através da aplicação de métricas de qualidade de processos, produtos e serviços.

Para que as medições sejam mais efetivas devem-se defini-las de acordo com objetivos específicos [1]. Uma abordagem bastante utilizada para alcançar esse fim é o Goal Question Metric (GQM).

No escopo desse trabalho, o Processo de atendimento de demandas de produtos de software da Empresa Brasileira de Pesquisa Agropecuária (Embrapa) é avaliado através da aplicação do GQM para identificar se esse processo está sendo utilizado e gerenciado adequadamente.

O documento está organizado nas seguintes seções: breve descrição do método GQM (seção 2), avaliação de um processo da Embrapa (seção 3), conclusões e sugestões de melhoria do processo (seção 4).

\section{Goal, Question, Metric}

O método GQM foi originalmente proposto por Basili (1992) citado por [1] para avaliar os defeitos de um conjunto de projetos da NASA Goddard Space Flight Center. Posteriormente, o uso do GQM foi expandido e tem sido adotado para medir e melhorar a qualidade em organizações de desenvolvimento de software.

De acordo com o mesmo autor, o modelo de medida proposto contém três níveis: Conceitual (Objetivo); Operacional (Questões) e Quantitativo (Métricas). O objetivo é definido para um objeto, e esse objeto pode ser um produto, um processo, ou um recurso utilizado por um processo. As questões definem um caminho para alcançar um determinado objetivo e tentam caracterizar o objeto de medida relacionado à qualidade. As métricas são definidas através de dados objetivos e subjetivos associados a cada questão de forma quantitativa.

O GQM compõe-se das seguintes fases: (i) planejamento, que envolve a seleção da aplicação a ser mensurada, definição, caracterização e planejamento do projeto; (ii) definição, os objetivos, questões, métricas e hipóteses são definidas e documentadas; (iii) coleta de dados, para atender as métricas definidas; e (iv) interpretação, na qual os dados coletados são analisados para identificar as respostas às questões [2].

\section{Avaliação de um processo da Embrapa}

A Embrapa compõe-se de trinta e sete unidades de pesquisa distribuídas nas regiões do país, três serviços especiais e doze unidades centrais na sede em Brasília e dois escritórios virtuais nos Estados Unidos e na França. Sua missão é "viabilizar soluções para o desenvolvimento sustentável do agronegócio brasileiro por meio de geração, adaptação e transferência de conhecimentos e tecnologia, em benefício da sociedade" [3].

A Tecnologia da Informação (TI) tem-se tornado estratégica para a Embrapa em relação ao desenvolvimento de produtos de software que transformam dados em informação e conhecimento, fundamentais nos processos de tomada de decisão e de atendimento ao cliente. Para facilitar o gerenciamento das novas demandas de produto de software, o Departamento de Tecnologia da Informação através do Grupo Corporativo de Desenvolvimento de Software, definiu e implantou o Processo de atendimento de demandas de produto de software em 2001. 
Esse processo é apoiado pelo Banco de Processos e Software que visa gerenciar os processos de negócio melhorados, as demandas e os produtos de software em uso na empresa. De forma geral, o processo compõe-se das seguintes atividades: i) Cadastrar demandas de produtos de software; ii) Analisar demandas de produtos de software e; iii) Atender as demandas de produtos de software. Essas atividades são divididas em tarefas que geram diversos documentos [4].

Desde sua implantação em 2001, o gerente não teve nenhum retorno sobre o uso desse processo e é importante avaliar se o mesmo está sendo realmente útil. Dessa forma, se faz necessário identificar a situação atual do processo através da aplicação de métricas de qualidade de processos.

A partir dessa necessidade e seguindo as fases propostas por [2], foi definido na fase de planejamento, a aplicação do GQM com o propósito de avaliar o processo de demanda de software da Embrapa. Estabeleceu-se uma equipe de trabalho de quatro membros, um deles definindo o processo e os restantes atuando como colaboradores para a avaliação. As demais fases estão descritas a seguir.

\subsection{Fase de definição}

O objetivo macro da análise do processo de demandas de produtos de software é avaliar a sua gestão e uso na Empresa. Ele foi definido com base no Objetivo estratégico "Gestão da informação" que compõe o Modelo Corporativo de Gestão Estratégica da Embrapa e tem como propósito melhorar o Processo de atendimento de demanda de produto de software, sob o ponto de vista do gestor do processo.

Após a definição do objetivo macro, foram analisados os indicadores do processo e definidos os seguintes objetivos específicos: 1) Melhorar a utilização do processo de atendimento de demandas de produto de software; 2) Melhorar a divulgação do processo de atendimento de demandas de produtos de software e; 3) Melhorar o nível de satisfação do usuário com relação ao processo de demanda de produtos de software.

Para o objetivo 1, foram definidas questões que caracterizam o objeto em estudo tais como: 1) Qual o percentual de demandas paradas; 2) Qual o percentual de demandas em atendimento; 3) Qual o percentual de demandas concluídas; 4) Qual a média de tempo gasto para atendimento das demandas concluídas e; 5) Qual o percentual de reclamações sobre as demandas paradas. Através destas questões foram definidas métricas para comparar os dados estimados com os dados reais identificados através da pesquisa. Desta forma, pode-se avaliar se o processo atende ao primeiro objetivo estipulado.

O objetivo 2 compõe-se das seguintes questões: 1) Qual o percentual de utilização do banco de software; 2) Como as demandas não cadastradas no banco estão sendo formalizadas e; 3) Qual o percentual de pessoas que desconhecem o processo e o banco de software. Através das respostas serão analisados os aspectos de divulgação do processo e se o mesmo atende ao segundo objetivo.

Para o objetivo 3, foram definidas as questões: 1) Qual o grau de dificuldade para utilização do processo e; 2) Em que nível a adoção deste processo facilitou o acompanhamento do atendimento da demanda. A partir das informações coletadas, será analisado o nível de satisfação em relação ao processo de atendimento de demandas.

Ao todo foram definidos 3 objetivos, 10 questões e 21 métricas. A tabela 1 apresenta como exemplo, o objetivo 1 e suas respectivas questões e métricas. 


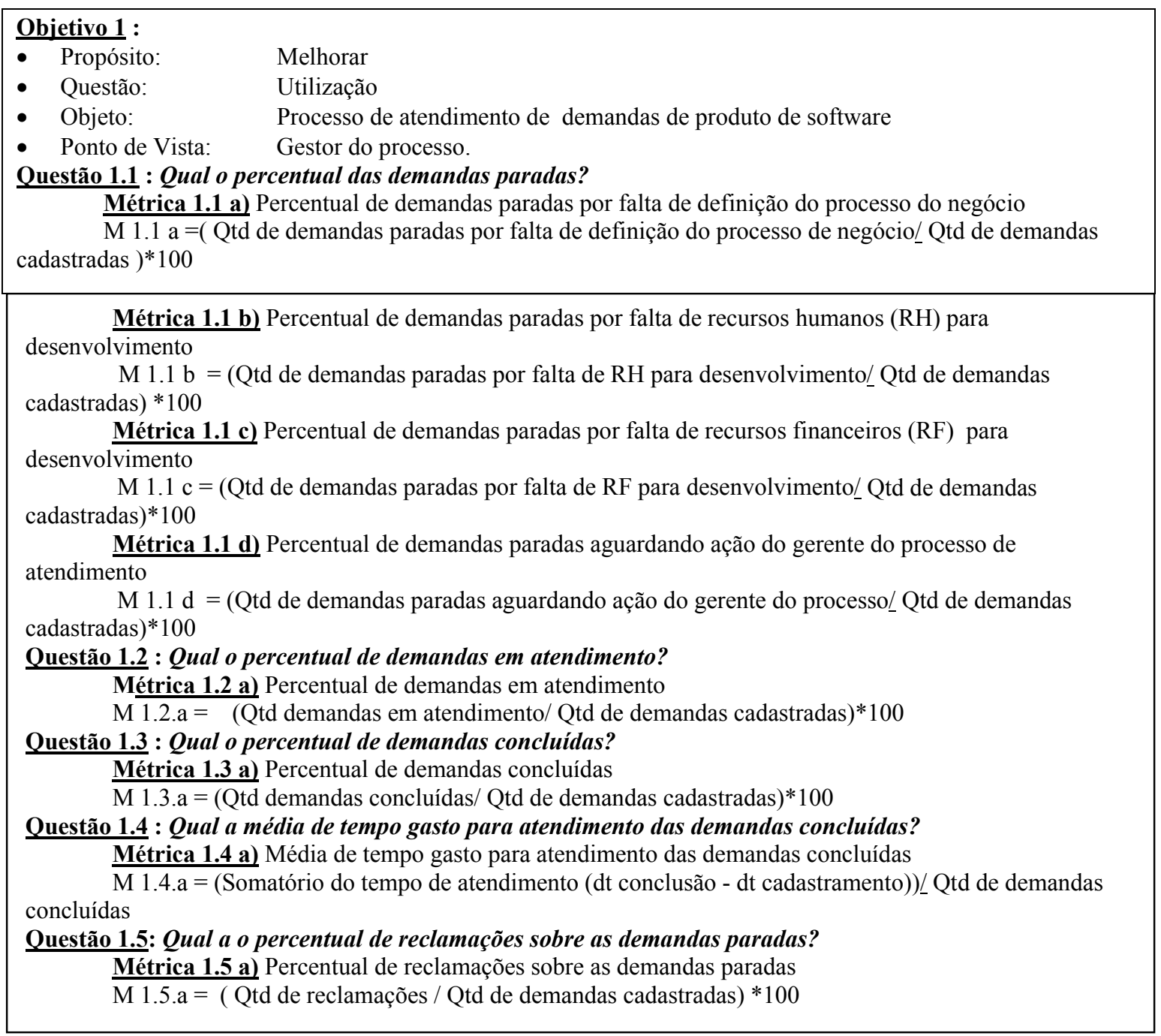

Tabela 1 - Objetivo 1: Melhorar a utilização do processo de atendimento de demandas de produto de software

\subsection{Fase de coleta de dados}

A coleta de dados foi realizada de acordo com os objetivos definidos. Para o objetivo 1: "Melhorar a utilização do processo de atendimento de demandas de produto de software" foram analisados os dados cadastrados no Banco de processos e software e realizada uma entrevista estruturada com o gestor do processo. Para os objetivos 2: "Melhorar a divulgação do processo de atendimento de demandas de desenvolvimento de software" e 3: "Melhorar o nível de satisfação do usuário com relação ao processo de demanda", os dados foram coletados através de questionário. A figura 1 mostra algumas questões do questionário.

O questionário foi enviado através de e-mail aos usuários cadastrados no Banco de processos e software (66 gerentes e 44 técnicos de TI). Foram obtidas 27 respostas sendo, 13 
de gerentes e 14 de técnicos de TI. O baixo número de respostas foi atribuído ao curto período da pesquisa e à decisão da gerência em não cobrar respostas visando identificar a espontaneidade no retorno da avaliação. Os resultados obtidos com a aplicação do GQM estão apresentados na tabela 2 .

\subsection{Fase de Interpretação dos dados}

A interpretação dos dados da aplicação do GQM será apresentada de acordo com os objetivos e as métricas definidas anteriormente.

\section{Figura 1 - Exemplo de algumas questões do questionário}

\begin{tabular}{|c|c|c|c|c|c|}
\hline 01. & $\square$ & Sim & 02 & $\square$ & Não \\
\hline \multicolumn{6}{|c|}{ 2. Caso afirmativo, você já cadastrou uma demanda de desenvolvimento de software no Banco de Processos e Software da Embrapa? } \\
\hline & \multirow{3}{*}{\multicolumn{2}{|c|}{$\begin{array}{l}\square \quad \operatorname{Sim} \\
\square \quad \text { Não houve demandas }\end{array}$}} & \multirow{2}{*}{$\begin{array}{l}02 \\
\square\end{array}$} & \multicolumn{2}{|c|}{$\square \quad$ Não. Por que? } \\
\hline & & & & \multicolumn{2}{|c|}{$\begin{array}{l}\text { Não sabia da existência do Banco de Processos e Software da } \\
\text { Embrapa }\end{array}$} \\
\hline & & & $\square$ & \multicolumn{2}{|r|}{$\begin{array}{l}\text { Não considera importante ou necessário o cadastro da demanda no } \\
\text { Banco de Processos e Software da Embrapa }\end{array}$} \\
\hline \multicolumn{6}{|c|}{ 3. Em caso afirmativo: } \\
\hline \multicolumn{6}{|c|}{ 3.a) Qual o grau de dificuldade para cadastrar a demanda de desenvolvimento de software no Banco de Processos e Software da Embrapa. } \\
\hline & $\begin{array}{l}\square \\
\square\end{array}$ & $\begin{array}{l}\text { Alta } \\
\text { Média }\end{array}$ & & $\begin{array}{l}\square \\
\square\end{array}$ & $\begin{array}{l}\text { Baixa } \\
\text { Nenhuma }\end{array}$ \\
\hline \multicolumn{6}{|c|}{ 3.b) Indique o grau de satisfação em relação ao atendimento da demanda cadastrada } \\
\hline & $\begin{array}{l}\square \\
\square\end{array}$ & $\begin{array}{l}\text { Muito Bom } \\
\text { Bom }\end{array}$ & $\begin{array}{l}03 \\
04\end{array}$ & $\begin{array}{l}\square \\
\square\end{array}$ & $\begin{array}{l}\text { Regular } \\
\text { A demanda não foi atendida }\end{array}$ \\
\hline & & & 05 & $\square$ & Outras. Especifique: \\
\hline
\end{tabular}

4. A adoção do processo de atendimento de demanda de produto de software facilitou o controle e o acompanhamento das demandas de software na sua UD?

\begin{tabular}{lll|lll} 
01. & $\square$ & Sim, muito. & 04 & $\square$ & Dificultou \\
02. & $\square$ & Sim, pouco & 05 & $\square$ & Não utilizou \\
03. & $\square$ & Não & 06 & $\square$ & Outras. Especifique:
\end{tabular}

5. Com que freqüência o desenvolvimento de software é solicitado em sua Unidade?

\begin{tabular}{lll|lll} 
01. & $\square$ & Anualmente & 03 & $\square$ & Sem periodicidade definida \\
02. & $\square$ & Semestralmente & 04 & $\square$ & Não solicita
\end{tabular}

6. Se sua demanda de desenvolvimento de software não é cadastrada no Banco de Processos e Software da Embrapa, qual o meio utilizado para fazer essa solicitação?

\begin{tabular}{lll|lll} 
01. & $\square$ & E-mail & 03 & $\square$ & Memorando \\
02. & $\square$ & Telefone & 04 & $\square$ & Sistema de ordem de Serviços (Ramal 4500) \\
& & & 05 & $\square$ & Outras. Especifique:
\end{tabular}




\begin{tabular}{|c|c|c|c|}
\hline & Questão & Métrica & Resultado \\
\hline \multirow{8}{*}{$\frac{2}{\sqrt[0]{0}}$} & \multirow[t]{4}{*}{$\begin{array}{l}\text { Questão 1.1 :Qual o } \\
\text { percentual das } \\
\text { demandas paradas? }\end{array}$} & $\begin{array}{l}\text { Métrica 1.1 a) Percentual de demandas paradas por falta de } \\
\text { definição de processo do negócio }\end{array}$ & $20 \%$ \\
\hline & & $\begin{array}{l}\text { Métrica 1.1 b) Percentual de demandas paradas por falta de } \\
\text { recursos humanos para o desenvolvimento }\end{array}$ & 0 \\
\hline & & $\begin{array}{l}\text { Métrica 1.1 c) Percentual de demandas paradas por falta de } \\
\text { recursos financeiros para desenvolvimento }\end{array}$ & 0 \\
\hline & & $\begin{array}{l}\text { Métrica 1.1 d) Percentual de demandas paradas aguardando } \\
\text { ação do gerente do processo }\end{array}$ & $40 \%$ \\
\hline & $\begin{array}{l}\text { Questão 1.2 : Qual o } \\
\text { percentual de demandas } \\
\text { em atendimento? }\end{array}$ & Métrica 1.2 a) Percentual de demandas em atendimento & $10 \%$ \\
\hline & $\begin{array}{l}\text { Questão 1.3 : Qual o } \\
\text { percentual de demandas } \\
\text { concluídas? }\end{array}$ & Métrica 1.3 a) Percentual de demandas concluídas & $\begin{array}{l}\text { Não existe nenhuma } \\
\text { demanda concluída }\end{array}$ \\
\hline & $\begin{array}{l}\text { Questão 1.4 :Qual a } \\
\text { média do tempo gasto } \\
\text { para atendimento das } \\
\text { demandas concluídas? }\end{array}$ & $\begin{array}{l}\text { Métrica 1.4 a) Média de tempo gasto para atendimento das } \\
\text { demandas concluídas }\end{array}$ & $\begin{array}{l}\text { Não existe nenhuma } \\
\text { demanda concluída }\end{array}$ \\
\hline & $\begin{array}{l}\text { Questão 1.5 :Qual o } \\
\text { percentual de } \\
\text { reclamações sobre as } \\
\text { demandas paradas? }\end{array}$ & $\begin{array}{l}\text { Métrica } 1.5 \text { a) } \\
\text { paradas }\end{array}$ & $\begin{array}{l}\text { Segundo o gerente não } \\
\text { houve nenhuma } \\
\text { reclamação }\end{array}$ \\
\hline \multirow{3}{*}{$\frac{\substack{0 \\
0}}{0}$} & $\begin{array}{l}\text { Questão 2.1 : Qual o } \\
\text { percentual de utilização } \\
\text { do banco de processos e } \\
\text { software? }\end{array}$ & $\begin{array}{l}\text { Métrica 2.1 a) Percentual de utilização do banco de } \\
\text { processos e software }\end{array}$ & $9 \%$ \\
\hline & $\begin{array}{l}\text { Questão 2.2 :Como as } \\
\text { demandas não } \\
\text { cadastradas no Banco } \\
\text { estão sendo } \\
\text { formalizadas? }\end{array}$ & $\begin{array}{l}\text { Métrica } 2.2 \text { a) Percentual de formalização utilizando e-mail } \\
\text { Métrica } 2.2 \text { b) } \\
\text { memorando }\end{array}$ & $\begin{array}{l}17 \% \\
23 \% \\
71 \%\end{array}$ \\
\hline & $\begin{array}{l}\text { Questão 2.3 :Qual o } \\
\text { percentual de pessoas } \\
\text { que desconhecem o } \\
\text { processo e o banco de } \\
\text { processos e software? }\end{array}$ & $\begin{array}{l}\text { Métrica } 2.3 \text { a) Percentual de técnicos de TI que desconhecem } \\
\text { o processo e o banco de processos e software } \\
\text { Métrica } 2.3 \text { b) Percentual de gerentes que desconhecem o } \\
\text { processo e o banco de software }\end{array}$ & $20 \%$ \\
\hline \multirow{2}{*}{$\frac{\sum_{0}^{\infty}}{0}$} & $\begin{array}{l}\text { Questão 3.1 : Qual o } \\
\text { grau de dificuldade } \\
\text { encontrada para } \\
\text { utilização do processo? }\end{array}$ & $\begin{array}{l}\text { Métrica 3.1 a) Percentual de baixa/nenhuma dificuldade } \\
\text { Métrica 3.1 b) Percentual de alta/média dificuldade }\end{array}$ & $\begin{array}{l}66 \% \\
25 \%\end{array}$ \\
\hline & $\begin{array}{l}\text { Questão } 3.2 \text { : Em que } \\
\text { nível o processo } \\
\text { facilitou o atendimento } \\
\text { da demanda? }\end{array}$ & $\begin{array}{l}\text { Métrica 3.2 a) Percentual do nível facilidade de controle e } \\
\text { acompanhamento- muito } \\
\text { Métrica } 3.2 \text { b) Percentual do nível - médio } \\
\text { Métrica } 3.2 \text { c) Percentual do nível - pouco } \\
\text { Métrica } 3.2 \text { d) Percentual do nível - nenhum }\end{array}$ & $\begin{array}{l}4 \% \\
0 \% \\
13 \% \\
4 \%\end{array}$ \\
\hline
\end{tabular}

Tabela 2 - Resultados da aplicação do GQM

\section{Objetivo 1: Melhorar a utilização do processo de atendimento de demandas de produto de software}

Constatou-se que apenas $10 \%$ das demandas cadastradas estão sendo atendidas, $40 \%$ estão aguardando ação gerencial e $20 \%$ estão esperando a definição do processo de negócio pelo gestor, um pré-requisito para o atendimento das demandas cadastradas. 
Nenhuma das demandas cadastradas teve o atendimento concluído até o momento. Apesar de $85 \%$ dos usuários respondentes considerarem esse processo importante, identificou-se que o mesmo é pouco utilizado, pois apenas $22 \%$ dos respondentes já cadastraram demandas. Para 19\% não houve demandas e para $12 \%$ não é solicitado software na unidade de pesquisa.

Segundo o gerente desse processo, nenhuma reclamação formal foi constatada em relação ao atendimento das demandas cadastradas no Banco de processos e software. Provavelmente, isto é conseqüência da baixa utilização ou desconhecimento do processo.

\section{Objetivo 2: Melhorar a divulgação do processo de atendimento das demandas de produto de software.}

Em relação ao conteúdo dos dados cadastrados no Banco de processos e software, identificou-se que $9 \%$ referem-se a demandas e $91 \%$ a software em uso, ou seja, o banco tem sido mais utilizado para controlar o software em uso que a demanda. Entre os 27 questionários respondidos, $81 \%$ dos usuários conhecem o processo, mas $70 \%$ ainda não o utilizaram. Para 12\% não há demandas.

Foi questionado sobre a periodicidade e a forma que a demanda é solicitada. Para $72 \%$ dos respondentes a periodicidade não é definida, para $8 \%$ é semestral e para $4 \%$ é anual e $12 \%$ não solicitam software. $17 \%$ formalizam as demandas por e-mail, $17 \%$ por telefone, $23,0 \%$ por memorando e para $13 \%$ as demandas são atendidas na própria unidade de pesquisa.

Pode-se constatar que o processo está muito pouco internalizado na empresa. Foi incluída uma questão no questionário sobre a melhor forma de divulgação do processo apesar da mesma não estar vinculada a nenhum dos objetivos definidos. Dentre os respondentes, $29 \%$ sugeriram a utilização de vídeo conferência, 20\% lista de discussão e 18\% através reuniões de Chefes de Unidades de pesquisas.

\section{Objetivo 3: Melhorar o nível de satisfação do processo de atendimento das demandas de produto de software}

Apesar do processo ser do conhecimento de $81 \%$ dos usuários que responderam o questionário e ser reconhecido como um processo de baixa/nenhuma dificuldade para $66 \% \mathrm{e}$ média/alta para $25 \%$, apenas $4 \%$ dos usuários consideram bom o nível de satisfação. Dentre os outros respondentes, $73 \%$ não responderam a questão e $19 \%$ não tiveram a demanda atendida.

Dentre os que utilizaram o processo, $13 \%$ consideram que o processo facilitou pouco o controle da demanda e para $4 \%$ não facilitou nada.

O gerente do processo ressaltou que há muitas dificuldades para priorização das demandas, poucos recursos humanos para o desenvolvimento interno e falta recursos financeiros para terceirizar o desenvolvimento. Ele também considera importante conscientizar os chefes de Unidades, gerentes de processos de negócio e os técnicos de TI sobre a necessidade do controle de demandas de produto de software para evitar re-trabalho e mau uso de recursos na Embrapa. 


\section{Conclusão}

Neste artigo, o Processo de atendimento de demandas de produtos de software da Embrapa foi avaliado para identificar se o mesmo está sendo utilizado e gerenciado adequadamente.

Nem todas as informações necessárias para a melhoria desse processo podem ser identificadas através da aplicação de GQM, mas os resultados obtidos representam no momento, um conjunto de indicadores que poderão ser utilizados para análise da situação atual do processo.

Diante dos resultados, é aconselhável que este processo seja melhorado e realizado um grande esforço para divulgá-lo e implantá-lo adequadamente na Empresa, pois os resultados mostraram que o mesmo não está bem internalizado e gerenciado. Mas, para que isso aconteça de forma satisfatória, recomenda-se também o envolvimento da Diretoria da Empresa, ou de um patrocinador, dos gestores de processos de negócio e da equipe corporativa de desenvolvimento de software para que num esforço conjunto, a idéia de gerenciar e controlar as demandas de software passe a ser vista como um benefício para a empresa.

A gestão das demandas cadastradas no Banco de processos e software deve ser rigorosamente realizada, pois esse processo é o ponto de partida para a implantação de todos os outros processos de software, a garantia da qualidade do produto de software e da satisfação do cliente e, principalmente, a diminuição de custos e recursos ao evitar que o mesmo processo de negócio seja automatizado por mais de uma Unidade de pesquisa.

Após todo este estudo, é importante ressaltar a identificação da técnica GQM como uma poderosa ferramenta de análise que pode trazer benefícios que, da forma tradicional, dificilmente poderiam ser alcançados. A aplicação desta técnica facilitou a identificação e definição dos objetivos, questões e métricas a serem utilizadas para medir o processo. Sua aplicação é recomendada em organização que ainda não tem cultura na implementação de planos de medição de processos e produtos de software.

\section{Referências Bibliográficas}

[1] BASILI. V.; CALDIERA, G.; ROMBACH, H. Goal Question Metric paradigm. In: Encyclopédia of Software Engineering. V. 2, 1994. p. $527-532$.

[2] SOLINGEN, R.; BERGHOUT, E. The Goal/Question/Metric method: a practical guide for quality improvement of software development. London: McGraw-Hill. 1999. 199p.

[3] EMBRAPA. Secretaria de Administração Estratégica. III Plano Diretor da Embrapa: realinhamento estratégico. 1999 - 2003. Brasília: Embrapa-SPI, 1998. 40 p.

[4] EMBRAPA. Departamento de Tecnologia da Informação. Relatório de descrição do processo: Atendimento de demandas de produtos de software da Embrapa. Departamento de Tecnologia da Informação. Brasília, 2001. 